\title{
ARTICLE
}

\section{Quality control to manage unsealed radioactive sources in nuclear medicine}

\author{
Sang Hyun Park ${ }^{*}$, Min Kyung Kim, Sang-eun Han, Hwe-in Kim, Min Chul Song and Jae Kwon Chang \\ Korea Institute of Nuclear Safety, 62 Gwahak-ro, Yuseong-gu, Daejeon, 34142, Korea
}

\begin{abstract}
According to the domestic and international regulatory framework of radiological protection, medical exposures of patients were regarded generally justified. However, there would be probability of discrepancy between prescribed dosage (or activity) and administered one during radiopharmaceutical delivery procedures. Due to inherent source of uncertainties, the discrepancies were reported up to \pm 10 to $20 \%$ for assuming well developed and good practices. So, the accurate assay of radiopharmaceuticals prior to administration is important process to assure that patients receive the correct prescribed dosage. In nuclear medicine practices, dose calibrators (radionuclide calibrators), usually well-type ionization chambers equipped with electrical circuit, have been used as the principal instrument to assay radiopharmaceutical dosage. Domestic regulation required licensee to maintain the patients' administered activity as prescribed by medical doctors. Based on the requirement, technical standards for activity measurements of unsealed radioactive sources in nuclear medicine practices and possession of measurement instruments were developed. Survey on current states of activity measurement in nuclear medicine practices, usage and quality control of dose calibrators and internationally recognized standards for quality control of dose calibrators were conducted to design appropriate regulatory approach.
\end{abstract}

\section{Keywords: medical exposure; nuclear medicine; activity measurement; dose calibrator;}

\section{Introduction}

In view of radiation protection and safety, medical uses of ionizing radiation have to be justified by weighing benefits of exposure against likelihood of the radiation detriment and considering alternative techniques that do not involve medical exposure. For each justified procedure, its exposure also should be optimized by keeping the exposure of patients to the minimum necessary to achieve the required medical objectives [1].

The accurate assay of activity of radiopharmaceuticals prior to administration is important process to assure that patients receive the correct prescribed dosage [2]. Dose calibrators (or radionuclide activity calibrators) have been used as the principal instruments to assay activity before administration. To measure the activity, the devices utilize ionization chambers of the well-type directly coupled to an appropriate electronic circuitry and a direct readout in units of activity [3]. With regard to activity measurements in nuclear medicine, it is recommended that verification of the appropriate calibration and conditions of operation of the dose calibrators should be included in framework of quality assurance (QA) program [4].

*Corresponding author. Email: shp@kins.re.kr
From the standpoint of the optimization, the levels of activity in radiopharmaceuticals to be administered clinically are governed primarily by the need to balance an optimal outcome (the effectiveness) and minimum exposure dose (the safety) [4]. In nuclear medicine practices, diagnostic and therapeutic dosages go through several stages from prescription to delivery. There are uncertainties in each stage. Typical sources of uncertainties are human error during dosage preparation, accuracy of dosage assay, delay time from preparation to administration (this is specific problem for short lived radionuclides, such as Tc-99m and F-18), and residual activities such as adsorption to syringe or vial wall. The uncertainties are ranged between \pm 5 and $10 \%$, and even up to $30 \%$ for the case of adsorption to vessel wall [2]. Considering these inherent uncertainties, in the United States, the Nuclear Regulatory Commission (NRC) requires that assayed dosages be within at least $\pm 20 \%$ of the prescribed dosage [5].

Domestic regulation of radiation safety control requires to maintain the patients' exposure dose or administered radioactivity as prescribed by medical doctors [6]. The relevant technical standards applied to radiation safety control in medical uses require licensee to establish documented quality control $(\mathrm{QC})$ program to ensure that all necessary procedures are developed and implemented to comply with the regulation [7]. However, the standards do not provide specific criteria 
for activity measurement before administration to patient undergone nuclear medicine treatments using unsealed radioactive sources.

In this study, regulatory provisions to manage unsealed radioactive sources in nuclear medicine practices and possession of measurement instruments with appropriate performance were proposed. The provisions were intended to support relevant domestic regulation for $\mathrm{QC}$ in medical uses of radiation. The provisions were developed based on international standards and current state of domestic licensees using unsealed radionuclides in nuclear medicine.

\section{Materials and methods}

\subsection{International requirements and guides of quality control of dose calibrators}

International Atomic Energy Agency (IAEA) required sources and instruments used for dosimetry of patients to be calibrated with internationally accepted or nationally accepted protocols and with appropriate traceability [1]. The agency provided detailed guides for these requirements in its technical report [4]. In the report, the agency recommended nuclear medicine practices to have more than one dose calibrator and to conduct acceptance test after installation. The calibrations of dose calibrators should be traceable to a secondary standards radioactivity laboratory (SSRL) or national metrology institute (NMI). Performance testing items with testing frequencies, simplified procedures and acceptance criteria were also provided to ensure appropriate operation and maintenance of dose calibrators.

NRC required that nuclear licensees using unsealed byproduct materials measure the activity of each dosage before medical use. For the measurement, NRC required licensees to have calibrated instrumentation in accordance with nationally recognized standards or the manufacturer's instructions [5].

Several international organizations and professional societies recommended standards and guidelines for calibration, usage and $\mathrm{QA} / \mathrm{QC}$ of dose calibrators $\left[2,3,8^{-11}\right]$. The standards provided testing items, testing methods and frequencies and acceptance criteria of each item. Criteria for accuracies of activity measurement using dose calibrator ranged from $\pm 5 \%$ to $\pm 10 \%$. Those criteria were listed in Table 1.

\subsection{Experiences of inter-laboratory comparison program of activity measurements with dose calibrators}

In the early 2000s, there was a steep increase in nuclear medicine and radiopharmaceuticals uses in Korea. So, to improve the accuracy of activity measurement in field instruments, Korea Food and Drug Administration (KFDA), the regulatory authority and Korea Research Institute of Standards and Science (KRISS), a national metrology institute (NMI) had managed a regular inter-laboratory comparison program of activity measurements with dose calibrators from 2002 to 2006 [12]. Among licensees, totally 71 nuclear medicine centers (hospitals) (79 dose calibrators) participated in the program voluntarily. Through the consecutive inter-laboratory comparison program, the measurement quality using field instruments was improved. The results showed that $61 \%$ and $65 \%$ of field measurements activities for I-131 and Tc-99m were within $\pm 5 \%$ of the correct value. These numbers increased up to $84 \%$ and $83 \%$ for I-131 and Tc-99m respectively, when a $\pm 10 \%$ limit was applied.

They also had conducted a research program on quality control of dose calibrators. Through the research program, they developed a guideline for QC of dose calibrators and technical report which backed up the guideline $[13,14]$. The guideline and technical report defined routine performance testing items with testing procedures and acceptance criteria. They also provided standard procedure of an activity measurement and correction of the activity with uncertainties.

Through the programs, they secured technical background and experiences to enhance the accuracy of activity measurement using dose calibrators. These would be supportive to implementation of the proposed regulation on QC of activity measurement in nuclear medicine practices.

Table 1. Criteria for accuracy of activity measurements using dose calibrators.

\begin{tabular}{ccc}
\hline Organizations $^{1)}$ & Accuracies $^{2)}$ & Remarks \\
\hline IAEA & $\pm 5 \%$ & $\begin{array}{c}1 \mathrm{MBq}<\text { Source activity } \\
<10 \mathrm{GBq}\end{array}$ \\
IEC, ANSI & $\pm 10 \%$ & $\begin{array}{c}\text { Source activity }>3.7 \mathrm{MBq} \\
\text { AAPM }\end{array}$ \\
& $\pm 10 \%$ & Diagnostic dosage \\
Therapeutic dosage \\
NPL & $\pm 5 \%$ & Gamma energy $>100 \mathrm{keV}$ \\
& $\pm 10 \%$ & Gamma energy $<100 \mathrm{keV}$ \\
KFDA & $\pm 10 \%$ & {$[15]^{3)}$} \\
\hline 1) IEC: International Electrotechnical Commission, \\
ANSI: American National Standards Institute, \\
AAPM: American Association of Physicists in Medicine, \\
NPL: National Physical Laboratory, \\
KFDA: Korea Food and Drug Administration. \\
2) The limits recommended by IAEA and NPL were calibrator \\
accuracies, and those by IEC, ANSI, AAPM, KFDA were \\
assay accuracies. \\
3) The standard which contained this criterion had been \\
withdrawn in 2009 (Notice of the KFDA No. 2009-23, May \\
2009).
\end{tabular}

\subsection{Survey on licensees which possess dose calibrators to assay radiopharmaceuticals}

Recently, Korea Institute of Nuclear Safety (KINS), surveyed present condition of usage and QC of dose calibrators $(2017.4 .13 \sim 5.30)$. Among all registered 211 licensees of using or producing unsealed sources, 204 licensees (185 hospitals with nuclear medicine practices and 26 organizations of production of unsealed sources with totally 336 dose calibrators) participated in the 
survey voluntarily. Main items of survey questionnaire were information of dose calibrators (numbers, manufacturer, model type, period of use, radioisotopes to measure) and $\mathrm{QA} / \mathrm{QC}$ program (possession of appropriate calibration sources, testing items and testing frequencies). The purpose of the survey was to estimate regulatory cost and to design appropriate regulatory approach in advance. The survey results are summarized in Figure 1.
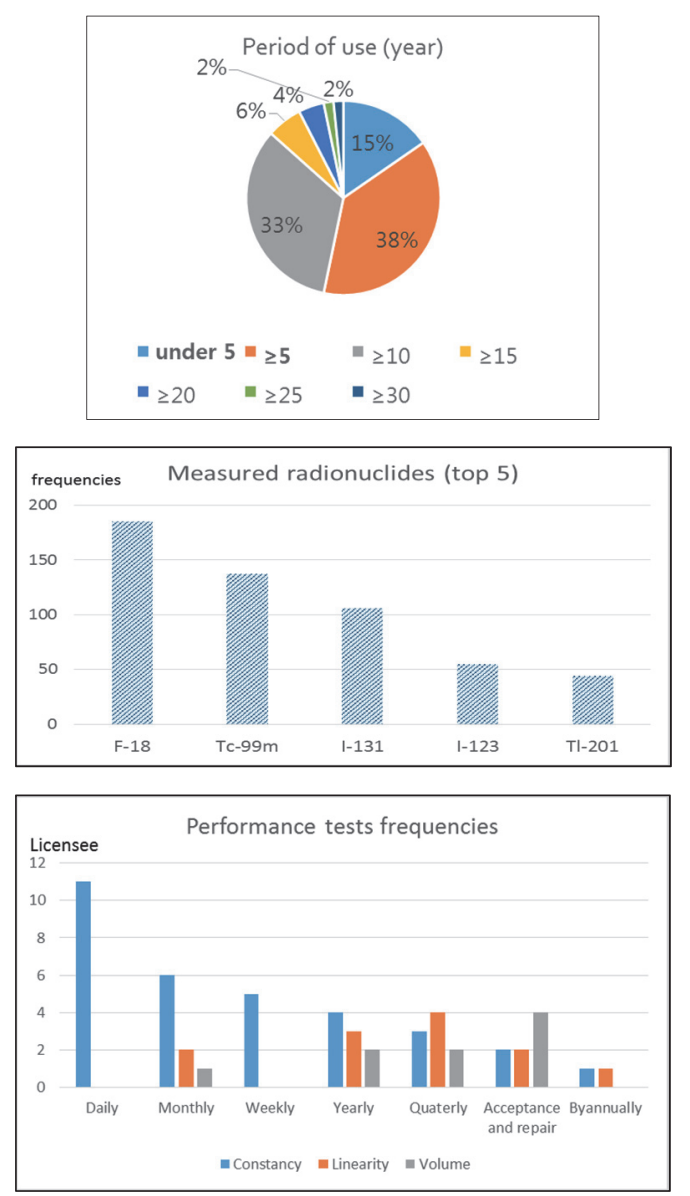

Figure 1. Survey results on QC of dose calibrators (Top: period of use, middle: top 5 radionuclides measured using dose calibrators, bottom: Performance tests frequencies of constancy, linearity and volume (or geometry)).

\section{Results and discussions}

\subsection{Current state of usage and QC of dose calibrators}

According to the survey results, almost licensees had more than one dose calibrator, except 13 licensees ( 8 licensees answered they had no dose calibrator and 5 licensees answered their dose calibrators were not available). For only $21 \%$ of licensees ( 33 hospitals and 13 licensees of production), QC of dose calibrators have been undertaken on a regular basis. Also only 52 licensees (37 hospitals and 15 manufacturers) possessed calibration sources which have metrological traceability to national standard, for example certified reference material (CRM) [16]. The calibration sources were mainly Ba-133, Co-57 and Cs-137. For Cs-137 (about $56 \%$ of total calibration sources), $52 \%$ of among them, the residual activities were not within the recommended activity range for calibration (i.e., 3.7 - 7.4 MBq) [10]. Though 46 licensees answered they have practiced QC of dose calibrators, for some routine performance test items, such as linearity, volume and activity correction, have been done by only 8 to 12 licensees. This implied that there were need to support licensees with education and training to enhance their understanding and to ensure appropriate level of QC undertaken to field instruments. Inter-laboratory comparison program of activity measurement would be a good solution to resolve this problem.

\subsection{Proposed regulatory provisions of quality control of nuclear medicine}

Technical standards for radiation safety control in medical use by the Nuclear Safety and Security Commission (NSSC), a regulatory authority, had a provision of QC mainly concerned with patient's exposure dose from radiation therapy [7]. Therefore, the provision should be revised to deal with exposure dose and administered activity as well. For this purpose, new provisions were proposed as follows;

- A licensee shall establish procedures to measure and determine the activity of each dosage before medical use.

- A licensee shall possess and use calibrated instruments to measure the activity of an unsealed radioactive source.

These provisions were proposed as minimum requirements of QC in nuclear medicine practices using unsealed sources. However, this new regulation would be active intervention in view of radiation protection. Because it focused on source term in exposure scenario to patients and medical staffs.

Though these provisions did not endorse specific standard or code, activity measurement procedures, QA/QC programs and calibration methods suggested by internationally and nationally recognized standards and guidelines would be applicable to comply with the new regulation.

\section{Conclusion}

In this study, regulatory provisions were proposed as minimum requirements to maintain a patient' administered radioactivity as prescribed by medical doctors. Because, considering current states of QC of activity measurement in nuclear medicine, licensees needed space to meet the regulation at an initial stage. As a regulatory practice, regulatory body required licensees to do basic QC procedures, such as daily constancy check. Their compliance will be examined through periodic inspection.

The purpose of the new regulation was to establish 
appropriate QA/QC for nuclear medicine practices using unsealed sources. QA/QC have been essential part to help licensees ensure successful optimization of protection and safety in medical uses of radiation [17]. It was expected that the new regulation would promote a prospective and iterative process of the optimization in nuclear medicine practices.

\section{References}

[1] International Atomic Energy Agency, Radiation protection and safety of radiation sources: international basic safety standards, IAEA Safety Series No. GSR Part 3, IAEA, (2014).

[2] American Association of Physicists in Medicine, The selection, use, calibration, and quality assurance of radionuclide calibrators used in nuclear medicine. AAPM Report of Task Group 181, AAPM, (2012).

[3] International Electrotechnical Commission, Nuclear medicine instrumentation - Routine tests Part 4: Radionuclide calibrators. IEC 61948-4(2006-11). IEC, (2005).

[4] International Atomic Energy Agency, Quality assurance of for radioactivity measurement in nuclear medicine, IAEA Technical Report Series No. 454, IAEA, (2006).

[5] U.S. Nuclear Regulatory Commission, Title 10 Code of Federal Regulations. Part 35 - Medical Use of Byproduct Material. (2007).

[6] Nuclear Safety and Security Commission, Regulations on Technical Standards for Radiation Safety Control Etc., (2016).

[7] Nuclear Safety and Security Commission, Technical standards for radiation safety control in medical use, Notice of the NSSC No. 2015-05 (radiation. 010), (2015).

[8] International Electrotechnical Commission, Medical Electrical Equipment: Radionuclide Calibrators - Particular Methods for Describing
Performance, IEC 61303(1994-10), IEC, (1994).

[9] International Electrotechnical Commission, Calibration and Usage of Ionization Chamber Systems for Assay of Radionuclides, IEC 61145(1992-05), IEC, (1992).

[10] American National Standards Institute, Calibration and Usage of "Dose Calibrator" Ionization Chambers for the Assay of Radionuclides, ANSI N42.13-2004 (Reaffirmation of ANSI N42.13-1986), ANSI, (2004).

[11] R. Gadd, M. Baker, K.S. Nijran, S. Owens, W. Thomas, M.J. Woods and F. Zananiri, Protocol for establishing and maintaining the calibration of medical radionuclide calibrators and their quality control, Measurement Good Practice Guide No. 93, National Physical Laboratory, (2006).

[12] G. Kim, H. Lee, H. Jeong and M.J. Woods, Comparison of radioactivity measurements with radionuclide calibrators in the Republic of Korea, Appl. Radiat. Isot. 63 (2005), pp. 201-205.

[13] Korea Food and Drug Administration, A Guideline for QC of Medical Radioactivity Calibrators, KFDA, (2008). [in Korean]

[14] Korea Research Institute of Standards and Sciences, Study on the Quality Control of Medical Radioactivity Calibrators, KRISS/IR-2006-071, KRISS, (2006). [in Korean]

[15] Korea Food and Drug Administration, Regulation on inter-comparison of radioactivity measurements, Notice of the KFDA No. 2004-3, (2004).

[16] Korea Research Institute of Standards and Sciences, 2015-2016 Certified Reference Materials Catalogue, KRISS/SP-2014-067, KRISS, (2014). [in Korean]

[17] International Atomic Energy Agency, Radiation protection and safety in medical uses of ionizing radiation, Draft Safety Guide (DS399), IAEA, (2016). 